\title{
Anemia en niños menores de tres años en la zona altoandina San Antonio - La Libertad
}

\section{Anemia in children under three years of age in the high Andean area San Antonio - La}

Libertad

\author{
Silvia Reyes ${ }^{1}$ (D)., Bibiana León ${ }^{2}$ iD y Allison Paredes ${ }^{3}$ (I).
}

\section{RESUMEN}

El estudio se realizó en el Puesto de Salud San Antonio de la Red de Salud Julcán-La Libertad con el objetivo de identificar los factores asociados a la anemia en niños menores de tres años en una zona altoandina del Perú. La investigación fue básica de diseño descriptivo, realizado en 55 niños seleccionados mediante un muestreo no probabilístico. Los datos se recolectaron con un cuestionario de factores asociados a la anemia con 19 ítems. La hemoglobina se midió con el hemoglobinómetro portátil (Hemocue HB 201). En el análisis univariado se utilizó la regresión logística y se calcularon los respectivos Odds ratio (OR) y sus intervalos de confianza de 95\%. Los resultados indican que existe mayor riesgo de padecer anemia cuando el niño no tiene seguro de salud (OR=1.875; IC:0.465 - 7.566), no acude a su control de crecimiento y desarrollo (CRED) (OR=2.6; IC:0.578 - 11.693), tiene bajo peso al nacer $(\mathrm{OR}=1.764$; IC:0.270 - 711.467), tiene parasitosis intestinal (OR=8.4; IC: 1.638 - 43.465), o cuya madre tiene más de tres hijos menores de tres años (OR =6.67, IC: $0.724-61.403)$. Se concluye que existen algunos factores de mayor riesgo relacionado a la presencia de anemia en niños.

Palabras clave: Anemia, factores de riesgo, cuidado del niño, salud del niño.

\begin{abstract}
The study was conducted in the San Antonio Health Post of the Julcán-La Libertad Health Network with the objective of identifying factors associated with anaemia in children under three years of age in a high Andean area of Peru. The research was a basic descriptive design, carried out on 55 children selected through non-probabilistic sampling. Data were collected using a 19-item questionnaire of factors associated with anaemia. Haemoglobin was measured with a portable haemoglobinometer (Hemocue HB 201). Logistic regression was used for univariate analysis and the respective odds ratios (OR) and their 95\% confidence intervals were calculated. The results indicate that there is a higher risk of anaemia when the child does not have health insurance (OR=1.875; CI:0.465 - 7.566), does not attend growth and development check-up (CRED) (OR=2.6; CI:0.578 - 11.693), has low birth weight (OR=2.6; CI:0.578 11. 693), has low birth weight (OR=1.764; CI:0.270 - 711.467), has intestinal parasitosis (OR=8.4; CI: 1.638 - 43.465), or whose mother has more than three children under the age of three (OR=6.67, CI: 0.724 - 61.403). It is concluded that there are some increased risk factors related to the presence of anaemia in children.
\end{abstract}

Keywords: Anemia, risk factor's, child care, child's health.

DOI: https://doi.org/10.37787/pakamuros-unj.v9i3.220

Recibido: 22/03/2020. Aceptado: 03/08/2021

* Autor para correspondencia

1. Universidad Nacional Santiago Antúnez de Mayolo, Perú. Email: reynaelizabet26@hotmail.com

2. Universidad Nacional Santiago Antúnez de Mayolo, Perú. Email bibianamarialh@hotmail.com

3. Universidad Nacional de Trujillo, Perú. Email: picali1496@hotmail.com 


\section{INTRODUCCIÓN}

La anemia es un problema de salud pública que afecta la salud y calidad de vida de niños y niñas en todo el mundo. Aproximadamente 1620 millones de personas sufre de anemia a nivel mundial, entre ellos el 47\% son niños menores de 5años (Uribe y Padilla, 2020). Esta enfermedad que ataca con frecuencia a la población infantil es común en países en vías de desarrollo y su origen está asociado a enfermedades como la diarrea, infecciones respiratorias, parasitosis y la deficiente alimentación (Aquino, 2021).

El país más afectado por anemia en Latinoamérica es Ecuador con el 37\% de niños escolares (Uribe y Padilla, 2020). En el Perú la anemia constituye un grave problema de salud, aun cuando los indicadores nacionales han disminuido, se ha reportado que en los últimos años el 40,1\% de niñas y niños sufre de anemia (6 a 35 meses de edad tiene anemia), el 49,0\% se encuentra en el área rural y el 36,7\% en el área urbana. En particular la región Ancash se registró 40,2\% de niños con anemia (INEI, 2019).

Es necesario unir esfuerzos para disminuir la anemia infantil, entre ellas la implementación de las políticas públicas a nivel nacional que incluye la articulación intersectorial, participación comunitaria y mejora de los servicios de salud (Unicef, 2019). Por esta razón, el Plan Nacional Para la Reducción y Prevención de la Anemia 2017 - 2021 considera la participación intrasectorial e intersectorial efectiva para disminuir al 20\% la anemia en niños menores de 3 años (MINSA, 2017). De igual modo se debe priorizar las intervenciones en el grupo más afectado como son los niños de 6 a 11 meses de edad (Zavaleta y Astete, 2017). Entre las intervenciones se consideran la lactancia materna exclusiva, suplementación con hierro, control de crecimiento y desarrollo, consejería, visita domiciliaria y control prenatal de la madre (MIDIS, 2018).

La anemia es producida por diversos factores como la ingesta insuficiente de hierro, prevalencia de enfermedades infecciosas (Ortiz, 2016). Los determinantes sociales que agudizan la presencia de anemia son la pobreza, condiciones de la vivienda, saneamiento básico, prácticas de higiene y el escaso conocimiento sobre la enfermedad entre otros (MIDIS, 2018). La anemia infantil repercute adversamente en el desarrollo psicomotor y a largo plazo en la esfera cognitiva, social y emocional del niño (Zavaleta y Astete, 2017). De allí la importancia de evitar la anemia en el primer año de vida para evitar consecuencias negativas en la salud física y emocional del niño.

En los estudios sobre anemia es necesario conocer los factores determinantes, entre ellos los de tipo social y cultural como la salubridad, hábitos de higiene, administración de multimicronutrientes y las prácticas de lactancia materna y alimentación (INEI, 2016). Además de los riesgos biológicos del niño, 
también se consideran situaciones como la anemia durante el embarazo, control prenatal y estado nutricional de la madre (Sguassero y col. 2018).

La anemia es un problema multicausal en la población infantil, así lo demostró Uribe y Padilla (2020), en Manabí Ecuador, que encontraron la presencia de anemia en el 11.29\% de la población en estudio asociada a la deficiencia de nutrientes y falta de micronutrientes. Al-kassab y Robles (2020), en Perú, observaron la prevalencia de 38,5\% de anemia infantil asociada significativamente a factores como lugar de residencia, región, altitud, quintil de riqueza, grado de instrucción de la madre y tipo de parto. De igual modo Mamani y Cajachagua (2019), en la localidad andina de Huanca-Perú reportaron 80\% de anemia en niños menores de 5 años, el promedio de Hb 11,8 g/dl, 90\% con parasitosis y 100\% padeció diarreas. La falta de agua segura predispone a los niños menores de 5 años a padecer anemia como consecuencia de la parasitosis intestinal y diarreas (Ortiz, 2016).

El estudio tuvo como objetivo identificar los factores asociados a la anemia en niños menores de tres años en una zona altoandina del Perú.

\section{MATERIALES Y MÉTODOS}

Investigación básica de diseño descriptivo realizada en el Puesto de Salud San Antonio en los niños que acuden a control de crecimiento y desarrollo durante los meses de enero a marzo del 2021. La muestra fue de 55 niños y niñas de 6 meses a 3 años seleccionados por muestreo no probabilístico. Los datos se recolectaron a través de un cuestionario de factores asociados a la anemia, instrumento elaborado por los autores, los mismos que fueron sometidos a la prueba de validez y confiablidad con el coeficiente alfa de Cronbach $(\alpha=.894)$ y evalúa los factores sociales, factores relacionados a la madre y factores relacionados al niño y su cuidado. La medición de la Hemoglobina (ajuste por altitud) se realizó con el hemoglobinómetro portátil (Hemocue HB 201), clasificando en: Normal (>11g/dl Hb, Anemia leve (10,9 - 10 g/dl Hb), Anemia moderada (9,9 - 7 g/dl Hb) y Anemia severa (<7 g/dl Hb). Los datos fueron procesados con el SPSS versión 25.0, para el análisis univariado se utilizó la regresión logística y se calcularon los respectivos Odds ratio (OR) y sus intervalos de confianza (IC) de 95\%. Para su ejecución se tuvo en consideración los principios éticos y la aprobación del Comité de Ética de la Universidad Nacional Santiago Antúnez de Mayolo. 


\section{RESULTADOS}

En la Tabla 1 se aprecia que existen dos factores de riesgo: El primero es la falta de seguro de salud, que indica que un niño que no cuenta con seguro de salud tiene aproximadamente 2 veces más riesgo de padecer de anemia, en comparación con un niño que si cuenta con seguro de salud. El segundo factor de riesgo fue la accesibilidad a controles del niño sano, que indica que si un niño no va a su control tiene aproximadamente 3 veces más riesgo de padecer anemia. Sin embargo, los intervalos de confianza (OR) nos indican que la anemia no está asociada a ninguna variable social (IC de OR contiene a 1, no existe asociación). Además, se observa que todas las viviendas (100\%) no cuentan con agua potable y del total de niños que presentan anemia el 26.9\% no están inscritos a un programa social del estado, en tanto que de los niños que no presentan anemia solo el 27.6\% no están inscritos a un programa social del estado, observándose que no existe diferencia significativa entre ambos grupos $(\mathrm{P}=.0 .956>0.05)$.

Tabla 1. Factores sociales según condición de anemia

\begin{tabular}{|c|c|c|c|c|c|c|c|c|}
\hline \multirow{2}{*}{ Variables } & \multicolumn{2}{|c|}{ Con anemia } & \multicolumn{2}{|c|}{ Sin anemia } & \multirow{2}{*}{ OR } & \multirow{2}{*}{\multicolumn{2}{|c|}{ IC $95 \%$}} & \multirow{2}{*}{ Valor $\mathbf{P}$} \\
\hline & $\mathbf{n}$ & $\%$ & $\mathbf{n}$ & $\%$ & & & & \\
\hline \multicolumn{9}{|l|}{ Abastecimiento de agua } \\
\hline No cuentan con agua potable & 29 & 100.0 & 26 & 100.0 & & & & \\
\hline Si cuentan con agua potable & 0 & 0.0 & 0 & 0.0 & & & & \\
\hline \multicolumn{9}{|l|}{ Tenencia de seguro de salud } \\
\hline No tienen seguro de salud & 6 & 23.1 & 4 & 13.8 & 1.875 & 0.465 & 7.566 & 0.373 \\
\hline Tienen seguro de salud & 20 & 76.9 & 25 & 86.2 & & & & \\
\hline \multicolumn{9}{|c|}{ Pertenece a un programa social del estado } \\
\hline No & 7 & 26.9 & 8 & 27.6 & 0.967 & 0.294 & 3.176 & 0.956 \\
\hline $\mathrm{Si}$ & 19 & 73.1 & 21 & 72.4 & & & & \\
\hline \multicolumn{9}{|c|}{ Accesibilidad a controles del niño sano } \\
\hline No Accesible & 6 & 23.1 & 3 & 10.3 & 2.6 & 0.578 & 11.693 & 0.203 \\
\hline Si accesible & 20 & 76.9 & 26 & 89.7 & & & & \\
\hline
\end{tabular}

La Tabla 2 muestra la existencia de dos factores de riesgo: El primero es el peso al nacer, que indica que si un niño tiene un peso al nacer menor a 2500 gr tiene aproximadamente 2 veces más riesgo de padecer de anemia (OR=1.764; IC:0.270 - 711.467) en comparación con un niño que pesa 2500grs o más. El segundo factor es la presencia de parasitosis intestinal que indica que si un niño presenta parasitosis 
intestinal tiene 8 veces más riesgo de padecer de anemia (OR=8.4; IC: 1.638 - 43.465) que un niño que no padece de parasitosis intestinal. Sin embargo, los intervalos de confianza del OR nos indican que solamente la presencia de parasitosis en el niño está asociada a la anemia. Con respecto a la presencia de infección respiratoria en el último mes, del total de niños con anemia el 26.9\% presentó infección respiratoria, en tanto que de los niños que no presentaron anemia el 34.5\% presento infección respiratoria, observándose que no existe diferencia significativa entre ambos grupos $(\mathrm{P}=.0 .55>0.05)$. Sobre la presencia de enfermedad diarreica, del total de niños con anemia el 15.4\% presento enfermedad diarreica, en tanto de los niños que no presentaron anemia el 31.0\% presentó enfermedad diarreica, siendo esta diferencia no significativa entre ambos grupos $(\mathrm{P}=0.173>0.05)$.

Tabla 2. Factores relacionados con el niño según condición de anemia

\begin{tabular}{|c|c|c|c|c|c|c|c|c|}
\hline \multirow{2}{*}{ Variables } & \multicolumn{2}{|c|}{ Con anemia } & \multicolumn{2}{|c|}{ Sin anemia } & \multirow{2}{*}{ OR } & \multirow{2}{*}{\multicolumn{2}{|c|}{ IC 95\% }} & \multirow{2}{*}{ Valor $\mathbf{P}$} \\
\hline & $\mathbf{n}$ & $\%$ & $\mathbf{n}$ & $\%$ & & & & \\
\hline \multicolumn{9}{|l|}{ Peso al nacer } \\
\hline Menos de $2500 \mathrm{gr}$ & 3 & 11.5 & 2 & 6.8 & 1.764 & 0.27 & 11.467 & 0.55 \\
\hline Más de 2500 gr. & 23 & 88.5 & 27 & 93.1 & & & & \\
\hline \multicolumn{9}{|c|}{$\begin{array}{l}\text { Presencia de infección respiratoria en } \\
\text { el último mes }\end{array}$} \\
\hline $\mathrm{Si}$ & 7 & 26.9 & 10 & 34.5 & 0.700 & 0.22 & 2.225 & 0.55 \\
\hline No & 19 & 73.1 & 19 & 65.5 & & & & \\
\hline \multicolumn{9}{|c|}{ Presencia de enfermedad diarreica } \\
\hline $\mathrm{Si}$ & 4 & 15.4 & 9 & 31.0 & 0.404 & 0.107 & 1.519 & 0.173 \\
\hline No & 22 & 84.6 & 20 & 69.0 & & & & \\
\hline \multicolumn{9}{|c|}{ Presencia de parasitosis intestinal } \\
\hline $\begin{array}{l}\mathrm{Si} \\
\text { No }\end{array}$ & $\begin{array}{l}10 \\
16\end{array}$ & $\begin{array}{l}38.5 \\
61.5\end{array}$ & $\begin{array}{c}2 \\
27\end{array}$ & $\begin{array}{c}6.9 \\
93.1\end{array}$ & 8.438 & 1.638 & 43.465 & 0.005 \\
\hline
\end{tabular}

En la Tabla 3 se aprecia la existencia de un factor de riesgo que es el número de hijos menores de tres años, que indica si una madre tiene tres o más hijos menores de tres años tiene aproximadamente 7 veces más de riesgo de padecer de anemia, en comparación con una madre que tiene menos de tres hijos menores de tres años. Sin embargo, los intervalos de confianza del OR nos indican que ninguna variable está asociada a la anemia (IC de OR contiene a 1, no existe asociación). Asimismo, se observa que 100\% de las madres realizó su control prenatal. Del total de madres de niños con anemia, 38.5\% presento anemia durante el embarazo y del total de madres de niños sin anemia, el 51.72\% no presento anemia 
durante el embarazo, por lo tanto, no existe diferencia significativa entre ambos grupos $(\mathrm{P}=0.319>0.05)$. Con respecto al parto institucional, las madres que no tuvieron un alumbramiento en un centro de salud fueron más frecuente en los hogares donde hay niños sin anemia (37.93\%) que en los hogares de niños con anemia (34.6\%), siendo esta diferencia no significativa $(\mathrm{P}=0.798>0.05)$. El 100\% de las madres, de niños que presentaron anemia, así como de niños que no presentaron anemia, no recibieron suplemento durante el embarazo.

Tabla 3. Factores relacionados con la madre

\begin{tabular}{|c|c|c|c|c|c|c|c|c|}
\hline \multirow{2}{*}{ Variables } & \multicolumn{2}{|c|}{ Con anemia } & \multicolumn{2}{|c|}{ Sin anemia } & \multirow{2}{*}{ OR } & \multirow{2}{*}{\multicolumn{2}{|c|}{ IC $95 \%$}} & \multirow{2}{*}{ Valor $\mathbf{P}$} \\
\hline & $\mathbf{n}$ & $\%$ & $\mathbf{n}$ & $\%$ & & & & \\
\hline No & 0 & 0 & 0 & 0 & & & & \\
\hline $\mathrm{Si}$ & 26 & 100 & 29 & 100 & & & & \\
\hline \multicolumn{9}{|c|}{ Presencia de anemia en el embarazo } \\
\hline $\mathrm{Si}$ & 10 & 38.5 & 15 & 51.72 & 0.583 & 0.199 & 1.708 & 0.319 \\
\hline No & 16 & 61.5 & 14 & 48.28 & & & & \\
\hline \multicolumn{9}{|c|}{ Parto Institucional } \\
\hline No & 9 & 34.6 & 11 & 37.93 & 0.866 & 0.288 & 2.609 & 0.798 \\
\hline $\mathrm{Si}$ & 17 & 65.4 & 18 & 62.07 & & & & \\
\hline \multicolumn{9}{|c|}{$\begin{array}{l}\text { Recibió suplemento de hierro en el } \\
\text { embarazo }\end{array}$} \\
\hline No & 26 & 100.0 & 29 & 100 & & & & \\
\hline $\mathrm{Si}$ & & 0.0 & & 0 & & & & \\
\hline \multicolumn{9}{|c|}{ Hijos menores de 3 años } \\
\hline 3 o más & 5 & 19.2 & 1 & 3.45 & 6.6 & 0.724 & 61.403 & 0.061 \\
\hline menos de 3 & 21 & 80.8 & 28 & 96.55 & & & & \\
\hline
\end{tabular}

En la Tabla 4 se observa que no existe ningún factor de riesgo relacionado a la presencia de anemia. Sin embargo, se encontró que 100\% de los niños recibió lactancia materna exclusiva (LME) hasta los 6 meses de edad. Del total de niños con anemia el 23.1\% no inicio la alimentación complementaria a los 6 meses de edad y del total de niños sin anemia solo el 10\% no inicio la alimentación complementaria a los 6 meses de edad; se observa que no existe diferencia significativa entre ambos grupos $(\mathrm{P}=0.204>0.05)$. El 76.9\% de las madres de niños con anemia, manifestaron que algunas veces da sulfato ferroso o hierro polimaltosado según la dosis indicada, en tanto que el $65.5 \%$ de las madres de niños sin anemia manifestaron que algunas veces da sulfato ferroso o hierro polimaltosado. Se observa que no existe diferencia significativa entre ambos grupos $(\mathrm{P}=0.352>0.05)$. Si se lavan las manos antes de preparar $\mathrm{y}$ 
dar los alimentos al niño, el 92.3\% de las madres de niños con anemia no existe diferencia significativa entre ambos grupos $(\mathrm{P}=0.730>0.05)$. Con respecto si mantiene a su hijo aseado y con ropa limpia, no se evidencio diferencia significativa.

Tabla 4: Factores relacionados al cuidado del niño

\begin{tabular}{|c|c|c|c|c|c|c|c|}
\hline \multirow{2}{*}{ Variables } & \multicolumn{2}{|c|}{ Con anemia } & \multicolumn{2}{|c|}{ Sin anemia } & \multirow{2}{*}{ OR } & \multirow{2}{*}{ IC $95 \%$} & \multirow{2}{*}{$\begin{array}{l}\text { Valor } \\
\mathbf{P}\end{array}$} \\
\hline & n & $\%$ & $\mathbf{n}$ & $\%$ & & & \\
\hline \multicolumn{8}{|c|}{$\begin{array}{l}\text { El niño recibió LME hasta los } 6 \\
\text { meses de edad }\end{array}$} \\
\hline No & 0 & 0.0 & 0 & 0 & & & \\
\hline $\mathrm{Si}$ & 26 & 100.0 & 29 & 100 & & & \\
\hline
\end{tabular}

La alimentación complementaría el inicio a los 6 meses

$\begin{array}{lcccccccc}\text { No } & 6 & 23.1 & 3 & 10.34 & 2.6 & 0.578 & 11.693 & 0.204 \\ \text { Si } & 20 & 76.9 & 26 & 89.66 & & & & \end{array}$

Su niño consume 3 comidas principales y dos refrigerios durante el día

\begin{tabular}{|c|c|c|c|c|c|c|c|c|}
\hline Casi siempre & 21 & 80.8 & 23 & 79.3 & 1.096 & 0.291 & 4.126 & 0.892 \\
\hline Siempre & 5 & 19.2 & 6 & 20.7 & & & & \\
\hline \multicolumn{9}{|c|}{$\begin{array}{l}\text { Da sulfato ferroso o hierro } \\
\text { polimaltosado según la dosis } \\
\text { indicada }\end{array}$} \\
\hline Algunas veces & 20 & 76.9 & 19 & 65.5 & 1.754 & 0.533 & 5.773 & \\
\hline Siempre & 6 & 23.1 & 10 & 34.5 & & & & 0.352 \\
\hline \multicolumn{9}{|c|}{$\begin{array}{l}\text { Se lava las manos antes de } \\
\text { preparar y dar los alimentos al } \\
\text { niño }\end{array}$} \\
\hline Casi siempre & 24 & 92.3 & 26 & 89.7 & 1.385 & 0.213 & 9.013 & 0.730 \\
\hline Siempre & 2 & 7.7 & 3 & 10.3 & & & & \\
\hline \multicolumn{9}{|c|}{$\begin{array}{l}\text { Mantiene a su niño aseado y con } \\
\text { ropa limpia }\end{array}$} \\
\hline Casi siempre & 23 & 88.5 & 22 & 75.9 & 2.439 & 0.559 & 10.646 & 0.213 \\
\hline Siempre & 3 & 11.5 & 7 & 24.1 & & & & \\
\hline
\end{tabular}

\section{DISCUSIÓN}

La presencia de anemia es más frecuente en los niños que no cuentan con seguro de salud, que no están inscritos a un programa social del estado y que no acuden programa de salud CRED. En ese sentido las 
desigualdades socioeconómicas y culturales permiten que se acentué la presencia de anemia en familias en condición de pobreza, de insalubridad, de malas prácticas de higiene y alimentación entre otras; por lo tanto, su abordaje debe ser intersectorial e intergubernamental para garantizar su efectiva intervención (MIDIS, 2018). La etapa de mayor vulnerabilidad de la persona es el primer año de vida donde el desarrollo del cerebro se consolida, de modo que, si hay déficit los efectos serán irreversibles (Zavaleta y Astete, 2017). Estos resultados difieren de Castro (2019), que encontraron anemia en 86\% de niños de 6 a 56 meses, de ellos 83,3\% están afiliados al Sistema Integral de Salud (SIS) y 12,5\% no tiene ningún tipo de seguro de salud. Por su parte Muñoz (2020), indica que el 21\% de niños con anemia todos tenían servicios básicos en su hogar.

Con respecto a los factores asociados con el niño se observa que el bajo peso al nacer y la parasitosis fue más frecuente en aquellos niños con anemia. Con respecto a la presencia de infección respiratoria y enfermedad diarreica el mayor porcentaje no fue determinante para la presencia de anemia. Estos resultados son similares a los reportes de Castro (2019), quienes observaron 86\% de niños de 6 a 56 meses con anemia, de ellos la prevalencia de enfermedad diarreica y respiratoria fue de 16,7 y 25\%. Sobre el parasitismo intestinal, Ruiz (2020), refieren que es una de las causas principales de anemia en los niños. Pero los datos encontrados difieren de Rivera y Arrunátegui (2019), quienes determinaron que los factores asociados a la anemia son la parasitosis intestinal y la presencia de infección respiratoria y diarreica las 2 últimas semanas, pues el valor normal de hemoglobina permite el buen funcionamiento del sistema inmunológico. Además, la velocidad de crecimiento aumenta las necesidades nutricionales de hierro y demás nutrientes, por lo que se debe asegurar su consumo desde antes del nacimiento, sobre todo en la gestación se debe cuidar a la madre con el control prenatal y administración de hierro para tener un recién nacido con peso normal (MINSA, 2017).

Asimismo, en relación a los factores asociados a la madre se encontró que todas las madres que realizaron su control prenatal sus hijos no presentaron anemia. Del total de madres que no tuvo anemia durante el embarazo el mayor porcentaje de los niños presentó anemia, las madres que no tuvieron el parto en un centro de salud fueron más frecuente en los hogares donde hay niños sin anemia. El total de las madres de niños que presentaron anemia, así como de niños que no presentaron anemia no recibieron suplemento durante el embarazo. De igual modo, las madres de niños con anemia tienen más de tres hijos menores de 3 años.

Estos resultados coinciden con el estudio de factores de riesgo de anemia donde el tipo de parto no es definitorio para anemia (RR: 1,2 [IC 9 5\%: 0,9- 1,6]) (Molina y Rens, 2020). Al-kassab A, et al. (2020), 
reportaron que los factores de mayor posibilidad de tener anemia son: quintil de riqueza, (pobre), 1/23; parto no institucionalizado 1/24, edad menor de 19 de la madre 1/34, instrucción primaria de la madre $1 / 25$, altitud $>=4000 \mathrm{msnm} 1 / 45$. La anemia genera un alto costo al estado, pues los efectos que produce pueden ser a corto y largo plazo, limitando la capacidad de las personas si esta ocurre en los primeros años de vida. Por lo tanto, es necesario acciones efectivas como la suplementación con hierro, promoción de la LME, evitar enfermedades en la infancia y mejorar la atención de salud (Zavaleta y Astete, 2017). No obstante Rivera y Arrunátegui (2019), reportaron que la anemia durante el embarazo aumenta más de 2.39 veces la prevalencia de anemia en niños menores de 3 años. Por otra parte, Villalba (2021), evidenciaron que las gestantes anémicas tienen 6 veces mayor probabilidad que sus hijos tengan bajo peso al nacer.

De igual forma sobre factores relacionados al cuidado del niño se encontró que todos recibieron LME hasta los 6 meses de edad. Sobre el inicio de la alimentación complementaria, consumo de alimentos durante el día, la administración de sulfato ferroso o hierro polimaltosado, el lavado de manos antes de preparar y dar los alimentos al niño, y si mantiene a su niño aseado y con ropa limpia se encontró que no existe diferencia significativa entre los niños con anemia y sin anemia.

Los resultados son similares a Castro (2019), quienes observaron que, de la prevalencia de niños con anemia, el 93,8\% tomaron leche materna y el 40\% tuvo LME. Con respecto al número de comidas 51,2\% consume tres veces/día y 26,8\% dos veces/día. De igual modo, Molina y Rens (2020), encontraron que $81,3 \%$ de madres de niños con anemia se lava las manos con agua y jabón y 44,4\% de niños no recibió suplemento de hierro. Muños (2020), reportaron 21\% de niños con anemia, de ellos 65\% recibió LME y 42\% recibió suplemento de hierro. También, Rivera (2019), observaron que la prevalencia de anemia en niños menores de 3 años tiene como factores de riesgo la falta de alimentación complementaria y el insuficiente consumo de alimentos ricos en hierro. En ese sentido es recomendable estrategias para la prevención de la anemia como talleres educativos y suplementación con hierro (Sguassero, 2018). De igual manera, Reyes et al. (2019), que estudiaron prevalencia de anemia en zonas rurales determinaron que luego de un programa de intervención se logró reducir los indicadores de anemia articulando el trabajo conjunto de la comunidad, universidad y sector salud. 


\section{CONCLUSIONES}

Entre los factores asociados a la anemia en niños menores de 3 años en una zona altoandina del Perú se encuentra mayor riesgo la falta de seguro de salud, el no acudir al CRED, el peso menor a 2500 gr al nacer, parasitosis intestinal y cuando la madre tiene más de tres hijos menores de tres años.

\section{REFERENCIAS BIBLIOGRÁFICAS}

Al-kassab-Córdova, A., Méndez-Guerra, C., Robles-Valcárcel, P. (2020). Factores sociodemográficos y nutricionales asociados a anemia en niños de 1 a 5 años en Perú. Rev Chil Nutr, 47(6), 925-932. http://dx.doi.org/10.4067/S0717-75182020000600925

Aquino, C. (2021). Anemia infantil en el Perú: un problema aún no resuelto. Revista Cubana de Pediatría, 93(1): e924. Recuperado de: http://www.revpediatria.sld.cu/index.php/ped/article/view/924

Castro, J, Chirinos, D. (2019). Prevalencia de anemia infantil y su asociación con factores socioeconómicos y productivos en una comunidad altoandina del Perú. Rev Esp Nutr Comunitaria, 25(3). Recuperado de: https://dialnet.unirioja.es/servlet/articulo?codigo=7137728

Fondo de las Naciones Unidas para la Infancia - Unicef. (2019). Lima Perú.

Instituto Nacional de Estadística e Informática. INEI. (2016). ENDES 2016 - Lima Perú.

Instituto Nacional de Estadística e Informática. INEI, (2019). ENDES 2019- Lima Perú.

Khan, L. (2018). Anemia in Childhood. Pediatr Ann, 47(2), 42-7. https://doi.org/10.3928/1938235920180129-01

Mamani, R., Alberca, A., Columbia, C., Cajachagua, M. (2019). Estrategias para disminuir diarreas parasitosis y anemia en menores de cinco años zona altoandina Perú. Horizonte sanitario, 18(3), 307-317. https://doi.org/10.19136/hs.a18n3.3019

Ministerio de Desarrollo e Inclusión social. MIDIS. (2018). Plan Multisectorial de Lucha contra la Anemia. Lima-Perú.

Ministerio de Salud del Perú. MINSA. (2017). Plan Nacional para la reducción y control de la anemia en el Peru: 2017-2021. Recuperado de: http://bvs.minsa.gob.pe/local/MINSA/4189.pdf

Molina, N., Rens, V. (2020). Anemia y déficit de hierro en lactantes de 6 a 12 meses de la ciudad de Necochea: prevalencia y determinantes. Arch Argent Pediatr, 118(3), 187-192. 
Muñoz, S., Naranjo, K. (2020). Factores de riesgo de anemia ferropénica en menores de 5 años hospitalizados Quito, 2020. (Tesis de pregrado). Universidad Nacional de Chimborazo, Ecuador. Recuperado de http://dspace.unach.edu.ec/handle/51000/7088

Ortiz, JS., Resende, M., Dinar, A. (2016). Impact of access to water and sanitation services on Education attainment. Water Resour Econ, 14(4), 31-43. https://doi.org/10.1016/j.wre.2015.11.002

Reyes, S., Contreras, A., Oyola, M. (2019). Anemia y desnutrición infantil en zonas rurales: impacto de una intervención integral a nivel comunitario. Journal of High Andean Research, 21(3): 205 214. https://doi.org/10.18271/ria.2019.478

Rivera, L., Arrunátegui, M. (2019). Anemia y factores asociados en niños menores de 3 años de Ayabaca - Piura 2019. (Tesis de pregrado). Universidad Privada Antenor Orrego. Recuperado de: https://hdl.handle.net/20.500.12759/6497

Ruiz, PA., Betancourt, SL. (2020). Sobre la anemia en las edades infantiles en el Ecuador: Causas e intervenciones correctivas y preventivas. Rev Cubana Aliment Nutr, 30(1): 218-235.

Sguassero, Y., Guerrero, M., y Romero, M. (2018). La visión de médicos pediatras de atención primaria de la salud sobre la anemia infantil y el suplemento con hierro. Archivos Argentinos de Pediatría, 116(1), 21-27. https://doi.org/10.5546/aap.2018.21

Uribe, V., Villacis, E., Padilla, A. (2020). Anemia por deficiencia de nutrientes en niños, niñas y adolescentes de la Zona Sur de Manabí. Pol. Con. 46(5), 309-327. Recuperado de: https://dialnet.unirioja.es/servlet/articulo?codigo=7518088

Villalva, J., Villena, J. (2021). Relación entre gestantes con anemia en edad materna de riesgo y bajo peso al nacer en un hospital de la seguridad social del Perú. Rev. Fac. Med. Hum, 21(1):101-107. https://doi.org/10.25176/RFMH.v21i1.3155

Zavaleta, N., y Astete L. (2017). Efecto de la anemia en el desarrollo infantil: consecuencias a largo plazo. Revista Peruana de Medicina Experimental y Salud Publica, 34(4): 716-722. https://doi.org/10.17843/rpmesp.2017.344.3251 